\title{
OP-0109 Protein profiling reveal SLC35b4 responds to glucose stimulation and acts through modulating molecular Hsp60 among others
}

Brigitte Wex, Rémi M. Safi, Gregory Antonios, Perla Z. Zgheib, Dania B. Awad, Firas H. Kobeissy, Rami A. Mahfouz, Marwan M. El-Sabban and Soha N. Yazbek*

*Medical Laboratory Sciences Program, Faculty of Health Sciences, American University of Beirut, Beirut, Lebanon

\section{Introduction}

Cumulative contribution of the identified diabetes genetic variants accounts for only $10 \%$ of total heritability. Our screen for diabetes genes in a unique mouse model (B6-A/J CSS) revealed:

- Over 143 genes may control diabetes predisposition

- Genes are context dependent with large non-additive effect size

Functional studies on the identified genes uncovered a novel role of SLC35b4

- In vivo alteration of hepatic gene expression of $S L C 35 \mathrm{~b} 4$ correlated with insulin resistance and gluconeogenesis

- In vitro alteration of $S L C 35 b 4 \mathrm{mRNA}$ altered gluconeogenesis

SLC35b4:

- Solute receptor gene on chromosome $7 \mathrm{q} 3$ expressed in most tissues

- Transports UDP-N-acetylglucosamine (UDP-GlcNAc) and UDPxylose (UDP-xyl)

- Function thought to mainly take place in the Golgi

- Literature linked it to several metabolic disorders in humans involved in obesity-induced diabetes

- SNP in the human SLC35b4 gene was associated with variation in body mass index (BMI), metabolic syndrome, fasting glucose, proinsulin levels, fat stores and obesity

\section{Aims}

We aimed to investigate $S L C 35 b 4$ 's role in the control of hepatic glucose production, an important mechanism for the response to insulin and for the alteration of glucose levels. This study investigated the localization of SLC35b4 and its role in the regulation of protein expression, and compared the differential expression between a knockdown of SLC35b4 and controls in HepG2.

\section{Methods}

SLC35b4's responsiveness to glucose, expression and localization were assayed using western blot and immunostaining. Localization was confirmed using DUO-Link PLA. 2D gel electrophoresis and MALDITOF were used to identify differentially expressed proteins between a knockdown of SLC35b4 and controls in HepG2 cells, and pathway analysis was performed using Elsevier's Pathway Studio version 10.0.

\section{Results}

Immunostaining for SLC35b4 in HepG2 cells showed that SLC35b4 is up-regulated in response to glucose stimulation.

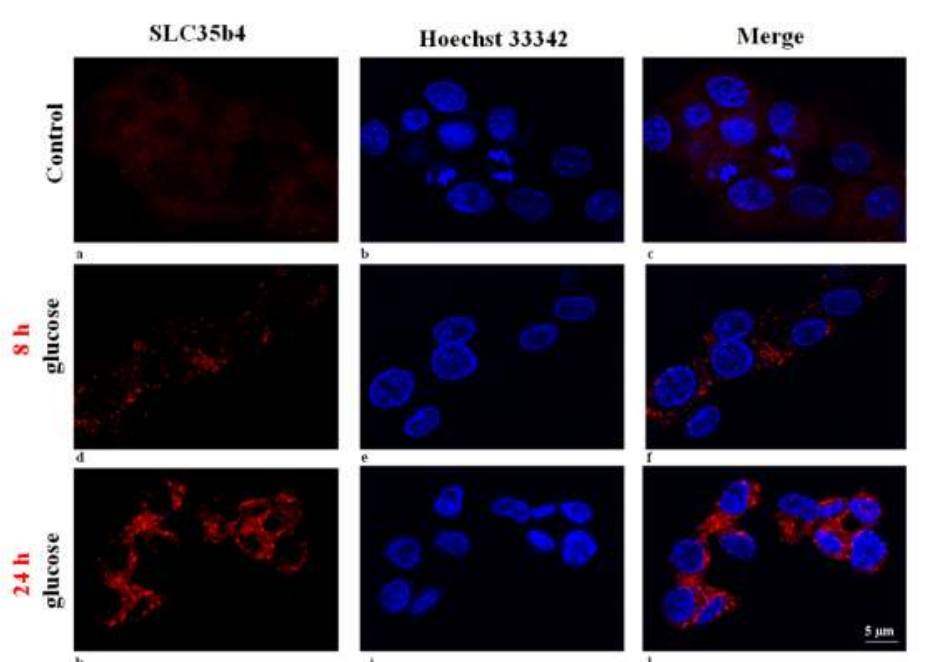

Western blots of SLC35b4 protein in response to glucose stimulation showed that SLC35b4 is up-regulated by $60 \%$ in glucose treated HepG2 cells
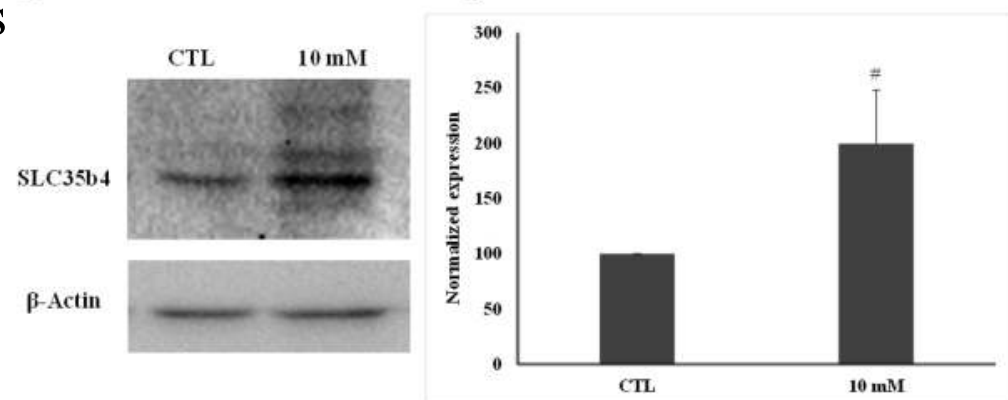

\section{Results $c n t^{\prime} d$}

Subcellular localization of SLC35b4 transporter in HepG2 cells by indirect immunofluorescence and DUO-Link PLA showed that SLC35b4 is widely spread in the Golgi apparatus and the ER.
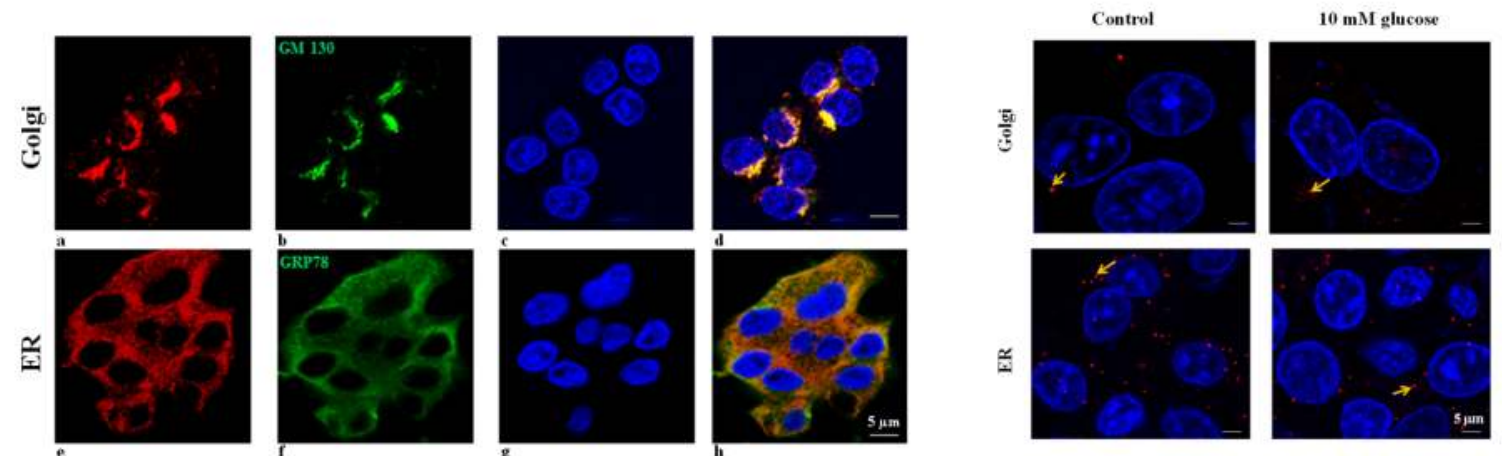

Immunostaining for SLC35b4 in normal liver tissue showed that SLC35b4 protein is expressed similar to HepG2 cells.
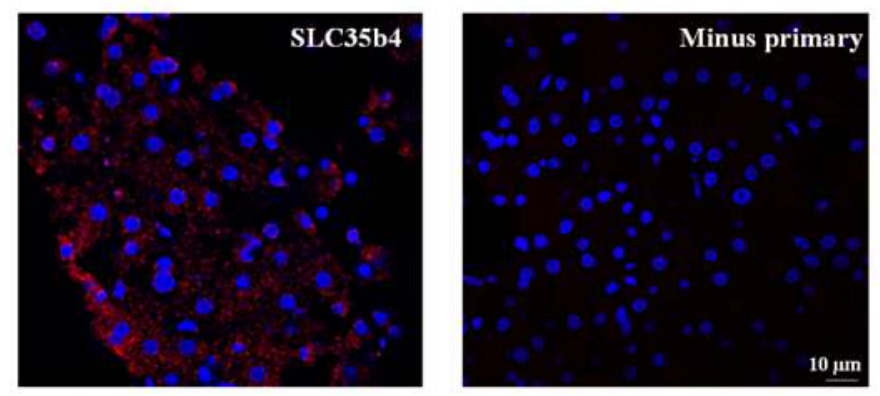

2D gel electrophoresis and MALDI-TOF showed that SLC35b4 knockdown in HepG2 cells altered the expression of multiple downstream proteins; four of which passed our stringent inclusion criteria and were successfully identified by MALDI (Hsp60, HSPA8, TUBA1A, Eno1). Panther pathway analysis revealed their connection to SLC35b4 and the insulin-glucose homeostasis pathway.

\section{Discussion and Conclusion}

- Literature has clearly linked Hsp60 to diabetes in humans, mice and beta cell cultures; mainly the increase in the levels of Hsp60 in both serum and saliva of diabetic patients.

- SLC35b4 Knockdown downregulated Hsp60 by 22 folds.

- Hsp60 being evidently modified by O-GlcNAcylation and the association of this modification with diabetic phenotype provides strong implication of the mechanism through which SLC35b4 could influence the downstream effect on Hsp60.

- Our report gives the first evidence of the causal effect that Hsp60 can play in hyperglycemia. Knockdown of SLC35b4 decreased the protein expression of Hsp60 which, as has been previously reported by our investigator, could have led to the increase in endogenous glucose production caused by SLC35b4 knockdown.

- We also found a significant down-regulation in HspA8 and ENO1 supporting the functional link of both to hyperglycemia.

- The $4^{\text {th }}$ gene that was significantly down regulated was TUBA1, a finding that implicates SLC35b4 in modulating O-GlcNAcylation.

We hypothesize that the uptake of UDP-GlcNAc by SLC35b4 carrier may have a negative feedback on the gluconeogenesis, leading to a decrease in glucose production. The finding that some of the downstream proteins are strongly modified by O-GlcNAcylation presents evidence to support this hypothesis

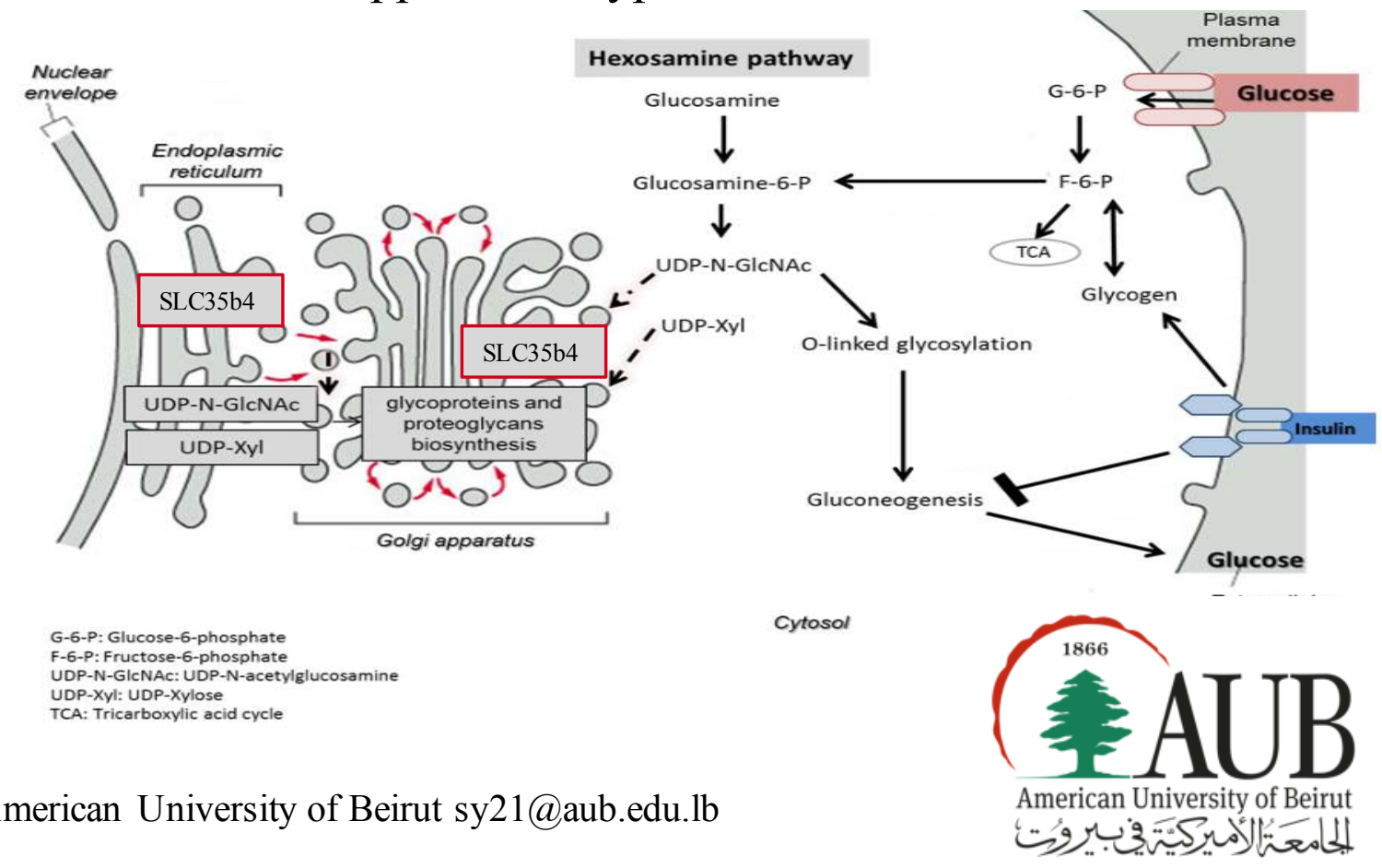

